\title{
Zur Entstehung von Literaturen zwischen zwei Welten
}

Gestatten Sie mir an dieser Stelle, einige kritische und selbstkritische Überlegungen zur Textsorte der Vorlesung und mehr noch zu einer Vorlesung über Literaturgeschichte anzustellen, die in Zusammenhang mit den soeben gestellten Fragen stehen! Denn dass es sich bei dieser Vorlesung über das 19. Jahrhundert zwischen zwei Welten um eine literarhistorische Vorlesung handelt, wird wohl niemand prinzipiell bestreiten wollen - auch nicht der Verfasser dieser Zeilen.

Zunächst einmal gilt es festzuhalten, dass bereits die Gattung oder Textsorte „Vorlesung“ eine für alle Beteiligten nicht nur schwierige und anspruchsvolle, sondern vor allem ebenso verantwortungs- wie widerspruchsvolle ist. Eine Vorlesung ist für mich persönlich - dies verberge ich Ihnen nicht - die schwierigste und zugleich großartigste der akademischen Übungen, denen sich ein Hochschullehrer bei der Anfertigung seiner nicht zuletzt auch didaktischen Materialien für die Studierenden unterziehen darf. Denn eine Vorlesung versucht - ganz allgemein gesprochen - in mehr oder minder ,traditioneller' Manier, ein bestimmtes Wissen über ein bestimmtes Wissensgebiet systematisch und didaktisch aufbereitet darzubieten. Dass es sich dabei im besten Falle um ein Wissen handelt, welches ein bestimmter Hochschullehrer oder eine bestimmte Hochschullehrerin im Verlaufe eines akademischen Lebens angesammelt hat, steht außer Frage. Und dies ist gleichbedeutend mit der Verpflichtung, für dieses dargebotene Wissen, das sich natürlich stets in unablässiger Bewegung und Veränderung befindet, auch zu einem gegebenen Zeitpunkt einzustehen.

Eine Vorlesung ist folglich ein offener Prozess; ein Buch hingegen besitzt einen Anfang und ein Ende, es birgt und verbürgt ein bestimmtes Wissen, welches bis zu einem genauen Zeitpunkt - in unserem Falle bis zum Beginn der zwanziger Jahre des 21. Jahrhunderts - erworben und formuliert werden konnte. Aus einer Vorlesung ein Buch und aus mehreren Vorlesungen Bücher zu machen, ist in der Tat weder risiko- noch folgenlos. Dieser Punkt markiert eine Schwierigkeit und Widersprüchlichkeit, die ich Ihnen nicht verbergen wollte.

Genau an dieser Stelle ergibt sich jedoch eine zweite Widersprüchlichkeit, sobald wir nicht mehr die Seite des Produzierenden, sondern der Rezipierenden genauer unter die Lupe nehmen. Denn gerade die mehr oder minder frontale Darbietungsweise verführt dazu, einer Vorlesung allenfalls zu ,folgen', sie also bloß zu konsumieren, ohne selbst am Stoff zu arbeiten, sich eigenständig mit bestimmten Gegenständen zu beschäftigen, hat doch bereits ein anderer an diesem Stoff gewirkt. 
Ein dritter Widerspruch resultiert in diesem Zusammenhang daraus, dass die Bezeichnung für diese Art der akademischen Veranstaltung selbst auf eine bestimmte Tätigkeit - eben das Vorlesen - verweist. Genau dieses findet aber hier nicht statt! Über mehr als zwei Jahrzehnte lang habe ich meine Vorlesungen nicht vorgelesen, sondern mündlich vorgetragen, wobei es stets im Hintergrund einen verschriftlichten Text gab. Auf diese Weise entstand aus einem schriftlichen Text in jeder Sitzung immer wieder etwas Unwiederholbares, das mich nicht selten selbst auch überraschte. So ging es mir in diesen Vorlesungen nie um einen Vortrag im Sinne einer abgeschlossenen Veranstaltung, sondern um ein Vortragen als offene Aktivität und Interaktion, der stets auch etwas Unvorhersehbares eignet. Die Vorlesung wird auf diese Weise einer nicht wiederholbaren Mündlichkeit anvertraut, die ganz von jener strengen Form der universitären Vorlesung abweicht, welche ich durchaus mit großer Bewunderung bei Erich Köhler an der Universität Freiburg kennenlernen durfte. Doch die unterschiedlichen Mündlichkeiten in den Vorlesungen eines Hugo Friedrich oder des Parapsychologen Hans Bender faszinierten mich schon als Studierenden und schlugen mich in ihren Bann.

Bei der schriftlichen Abfassung jenes Vorlesungstextes, den Sie gerade hören beziehungsweise lesen, habe ich mich sehr wohl von unterschiedlichen Formen der Mündlichkeit mit ihren integrierten Exkursen oder Digressionen leiten lassen. Es handelt sich freilich aus evidenten didaktischen Gründen um eine Mündlichkeit, die in erster Linie auf Verständlichkeit und Verstehen abzielt. Damit verändern sich in gewisser Weise unsere Rollen. Lassen Sie mich das kurz erläutern!

In der traditionellen Vorlesung, für die ich Erich Köhlers brillante AudimaxVeranstaltungen einmal stellvertretend nennen darf, wurde schriftlich Ausformuliertes vorgelesen, das dann von den Zuhörerinnen und Zuhörern wiederum in ein Wissen in mündlicher Form umgewandelt wurde, um in einem zweiten Schritt in Form von Mitschriften wiederum in eine andere schriftliche Form zurückverwandelt $\mathrm{zu}$ werden.

Die mündliche Form des Vortragens auf Basis eines schriftlichen Textes übernimmt damit in gewisser Weise Ihren Part: die Überführung in eine hoffentlich möglichst verständliche Mündlichkeit. Was aber ist dann bei diesem Wechselspiel Ihre Aufgabe? Nun: lesen, lesen, lesen! Aber auch das Zuhören, und deshalb beabsichtige ich - neben anderen Gründen - auch, immer wieder Akustisches einzublenden, etwa aufgesprochene Zitate oder akustische Zeichen oder Musikstücke aus dem Kontext der jeweils behandelten Epoche. Dies soll den Ablauf der Vorlesung noch lebendiger gestalten. Freilich kann ich Ihnen zum 19. Jahrhundert keine Ausschnitte aus Autorenlesungen oder Interviews mit den von uns behandelten Schriftstellerinnen und Schriftstellern bieten. Ich hoffe, Sie sehen mir das nach und akzeptieren auch die zahlreichen Illustrationen und Gemälde, welche die Vorlesungen bereichern sollen, ohne Sie vom Wesentlichen abzulenken. 
Gleichviel, ob wir von einer mündlichen oder einer schriftlichen Form dieser Vorlesung ausgehen: Sie entfaltet auf narrative Weise eine Geschichte. Der Verfasser einer Vorlesung zum Thema Literaturgeschichte ist folglich ein Geschichtenerzähler. Wir haben einleitend bereits die Tatsache behandelt, dass sich der Kollektivsingular der „Geschichte“ geschichtlich im letzten Drittel des 18. Jahrhunderts bildete und dies etwas zu tun hatte mit der Herausbildung eines modernen Begriffs zukunftsoffener Geschichte, die sich zunehmend einer zyklisch angelegten Geschichtsvorstellung entzog. Eine Vielzahl von Geschichten, die erzählt werden können, fließt in die Geschichte ein, welche sich als solche aber stets in Bewegung befindet und ständigen Veränderungen unterliegt. Ebenso die unendlich vielen Geschichten, die man erzählen hört, als auch die Geschichte selbst müssen narrativ aufgearbeitet und folglich erzählt werden.

Auch die Rezipientin oder der Rezipient, die einen bestimmten Text - oder eine Vorlesung - aufnehmen, tun dies: Sie erzählen sich eine Geschichte oder sie erzählen diese Geschichte - je nach vorhandenen Wissensbeständen - in veränderter Form nach. Der US-amerikanische Literaturtheoretiker Brian McHale hat erzählt, dass er eines Abends zur Einsicht gelangt sei, dass er nicht nur seinen Kindern Betthupferl-Geschichten erzähle, sondern auch seinen Studierenden, wenn er ihnen in seinen Vorlesungen literarische Entwicklungen und Probleme präsentiere. ${ }^{1}$ Daraus ergibt sich der notwendig narrative Charakter unserer Veranstaltung: Ich erzähle Ihnen eine Geschichte, die freilich recht lang werden wird, denn sie geht erst am Ende dieses Semesters zu Ende. Ich erzähle Sie ihnen gleichsam in Fortsetzungen und immer zuverlässig zur selben Zeit.

Das mag Ihnen jetzt so vorkommen wie jene Figur eines Monsieur Jourdain, der sich in Molières Komödie Le Bourgeois gentilhomme plötzlich der Tatsache bewusst wird, dass er die ganze Zeit schon in Prosa spricht. Gewiss! Aber wenn wir uns der Tatsache bewusst werden, dass wir eine Geschichte erzählen, dann können wir auch leichter die narrativen Dimensionen unseres Tuns vergegenwärtigen und narratologisch fundierte Analysen all jener Teil-Geschichten vorlegen, die ich Ihnen im Verlaufe unserer Vorlesung erzähle. Wir reflektieren damit unser eigenes Tun, und eben dies ist die Grundlage von Wissenschaft. Das ist nicht mehr als ein erster Schritt, um aus Naivität herauszukommen, aber es ist ein wichtiger Schritt.

Wenn Sie im Verlauf dieser Veranstaltung gut aufpassen, werden Ihnen eine Vielzahl guter Erzähler, Erzählinstanzen und Erzählerfiguren begegnen. Einige dieser Geschichtenerzähler kennen Sie schon, und auch manche ihrer Erzähler.

1 Vgl. McHale, Brian: Postmodernist Fiction. London: Methuen 1987; sowie (deres.): Constructing Postmodernism. London - New York: routledge 1992. 
Sie haben bereits Erfahrungen mit den Texten von Bernardin de Saint-Pierre, Heinrich von Kleist oder Chateaubriand gemacht und haben schon zwischen Erzählern und Erzählfiguren auf Ebene einer Rahmenerzählung wie einer Binnenerzählung unterschieden. Erzählformen sind so alt wie die Literaturen der Welt. Aber das 19. Jahrhundert ist erzähltechnisch gesehen die Epoche, in welcher sich die allermeisten narrativen Konventionen etablierten und sich jene Erzählformen und Erzählnormen herausbildeten, die in gewisser Weise noch immer die Grundstrukturen unseres Erzählens im 21. Jahrhundert bilden.

Ob ich selbst auch ein guter Erzähler sein werde, können allein Sie als Zuhörerinnen und Zuhörer, als Leserinnen und Leser beurteilen. Immerhin aber kann ich Ihnen einiges über Erzähler und das von ihnen Erzählte sagen. Zugleich jedoch ist eines deutlich: Es handelt sich bei unserer Vorlesung - und dies ist ein vierter Widerspruch - um eine Überblicksvorlesung, die Ihnen einen Überblick über den behandelten literaturgeschichtlichen Stoff verschaffen möge. Die Veranstaltung soll Orientierungshilfe für das Studium auf inhaltlicher Ebene sein und - wenn möglich - einige der wichtigsten und repräsentativsten Werke der romanischen Literaturen der Welt im 19. Jahrhundert vorstellen.

Doch soll zugleich die Überblicksveranstaltung nicht zum Überfliegen der verschiedensten Texte, Probleme und Themenbereiche einladen. Wir werden uns auch im weiteren Verlauf der Vorlesung auf bestimmte Texte beziehungsweise Werke konzentrieren, die im Sinne der Geschichte, die hier erzählt wird, als repräsentativ erscheinen und herausragende Facetten und Aspekte einer literarischen Entwicklung demonstrieren, die für diese Geschichte relevant sind. Ungeachtet des Überblickscharakters werde ich Ihnen eine Geschichte erzählen, die meine Geschichte der Literaturen des 19. Jahrhunderts ist und ihren Schwerpunkt in der Zusammenschau beider Seiten des Atlantik findet - und dies nicht allein, weil ich ein solches Vorgehen für höchst innovativ halte. Ich tue dies auch, weil aus meiner Sicht diese Geschichte genau so, also in der wechselseitigen Verklammerung unterschiedlichster Literaturen jenseits des Nationalen und jenseits nationalgeschichtlicher Festschreibungen grenzüberschreitend, transareal und transkulturell erzählt werden muss.

All diese Überlegungen beinhalten, dass es sich bei dieser Literaturgeschichte - wie bei allen Literaturgeschichten - um eine bewusste Konstruktion handelt und die Literaturgeschichte als solche folglich ein Konstrukt ist dessen, der sie vorträgt. Dabei versichert sich der wissenschaftliche Geschichtenerzähler immer wieder seiner genau überprüfbaren Bezugstexte oder historischen Quellen, aber auch der Übereinstimmung mit und der klaren Diskrepanz gegenüber Wissenschaftlerinnen und Wissenschaftlern, die über ähnliche Fragestellungen bereits gearbeitet haben. Dies unterscheidet wissenschaftliche Theorie von Ideologie: Erstere ist jederzeit überprüfbar und argumentativ, faktisch und 
methodologisch verifizier- oder falsifizierbar. ${ }^{2}$ Denn selbstverständlich basiert jegliche Konstruktion auf vorherigen Vermessungen sowie auf Methoden und Konzepten, die - wie eben gerade das Konzept und die Anwendung des Terms „Transkulturation“ - aus der vorangehenden Forschungsliteratur kritisch entwickelt wurden.

Jede Literaturgeschichte - ganz gleich, welchem Objekt sie sich widmet beruht auf artifiziellen, künstlichen Setzungen: Zum einen wird festgesetzt, was für eine bestimmte Literaturgeschichte oder Überblicksvorlesung relevant ist, was also behandelt wird, und was draußen vor der Tür bleibt. Zum anderen wird festgelegt, welcher Bereich innerhalb literaturgeschichtlicher Fragestellung als kohärent aufgefasst wird. In der Literaturgeschichtsschreibung hat man sich oft aus nationalen oder gar nationalistischen Gründen - auf die Geschichte von Nationalliteraturen festgelegt, auch wenn es die Nation als solche über lange Zeiträume noch gar nicht gab. Es genügt, dabei etwa an die deutsche oder kubanische Nation und Literaturgeschichte zu denken, wo in beiden Fällen die ,Kulturnation“ der staatlich-politischen Nation vorausging.

Wenn wir uns etwa mit europäischen Literaturen oder den Literaturen Lateinamerikas beschäftigen, so verlassen wir ganz bewusst das enge Terrain nationalliterarischer Historiographie. Wir bekommen es dann simultan mit mehreren Literaturen zu tun, welche miteinander in einem wechselseitigen Austauschprozess stehen, der von einer national ausgerichteten Literaturgeschichtsschreibung $\mathrm{zu}$ einem beträchtlichen Teil übersehen wird. Beschäftigen wir uns mit den Literaturen Europas, so müssen wir die jeweiligen Grenzen des Kontinents bedenken und dürfen uns nicht etwa darauf verlassen, wer innerhalb der Europäischen Union ist. Widmen wir uns den lateinamerikanischen Literaturen, so sind etwa die anglophonen, holländischen oder indischen Literaturen der Karibik ebenso ausgeschlossen wie die englischsprachige Literatur der USA, nicht aber die Literaturen der Latinas oder Latinos. Sie sehen, dass die jeweiligen Ausschlussprozesse wichtig sind, um die spezifischen Koordinaten des Konstruktionsprozesses abzustecken.

Würden wir einen Term wie die „Literaturen Hispanoamerikas“ wählen, so wären die frankophonen Literaturen Kanadas oder der Antillen ebenso ausgeschlossen wie die portugiesischsprachigen Brasiliens, welche wiederum bei der Verwendung des Begriffs der Literaturen Iberoamerikas miteingeschlossen blieben. Unterschiedliche Trennschärfen wären aber auch bei einer deutschen Literaturgeschichte $\mathrm{zu}$ beachten, die sich als nationales Konstrukt erheblich von

2 Vgl. hierzu Zima, Peter V.: Ideologie und Theorie. Eine Diskurskritik. Tübingen: A. Francke Verlag 1989. 
einer Literaturgeschichte deutschsprachiger Literaturen unterscheiden müsste, wobei es auch auf diesem Gebiet spannende Entwicklungen gibt, wie Sandra Richters Weltgeschichte der deutschsprachigen Literatur belegen mag. ${ }^{3}$ Sie sehen: Auch die germanistische Literaturgeschichtsschreibung wird im Rahmen ihrer Möglichkeiten global.

Wenn wir den Bereich einer einzigen Nationalliteratur verlassen und uns zugleich mit mehreren und verschiedensprachigen auseinandersetzen, dann wird klar, dass wir komparatistisch arbeiten - also zwischen verschiedenen Nationalliteraturen vergleichend. Es wird allerdings noch zu zeigen sein, dass aus ganz spezifischen historischen und kulturellen Gründen weder der Begriff „Nationalliteratur“ noch jener der „Komparatistik“ oder der „Vergleichenden Literaturwissenschaft" ganz auf die Verhältnisse in Lateinamerika passt beziehungsweise angepasst ist. Zwischen dem Konzept der Nationalliteratur und jenem der lateinamerikanischen Literaturen wurde versucht, mit der Unterscheidung zwischen verschiedenen literarischen Regionen des Subkontinents eine Art Zwischenebene zu schaffen, welche die plurale Vielfalt und Unterschiedlichkeit dieser Literaturen und Kulturen zum Ausdruck bringt. Ich werde auf dieses Konzept, das vor allem von Alejandro Losada ${ }^{4}$ vorangetrieben und vertreten wurde, immer einmal wieder zurückkommen. Auch hier werden wir rasch an die Grenzen konzeptueller Tragfähigkeit stoßen. Dennoch scheint es mir wichtig, derartige Vorstellungen in unsere Vorlesung zu integrieren und etwa zwischen kulturell-literarischen Areas wie dem Andenraum oder dem Cono Sur, Brasilien oder der Karibik zum gegebenen Zeitpunkt zu unterscheiden.

Adalbert Dessau, der Begründer des Rostocker Lateinamerika-Zentrums in der damaligen Deutschen Demokratischen Republik, sprach einmal von der Dialektik zwischen dem Nationalen, dem Kontinentalen und dem Internationalen. ${ }^{5}$ Das waren damals erste Anfänge einer transarealen Konzeption der lateinamerikanischen Literaturen. Quer dazu liegen die - je nach Stand der Theoriebildung fünf verschiedenen Regionen oder kulturelle Areas, die der damals in West-Berlin arbeitende Alejandro Losada unterschied: Diese fünf Teilregionen sind Mexiko, Mittelamerika, Brasilien, der Cono Sur und der pazifische Andenraum. Die kulturell hochkomplexe Welt der Karibik lässt sich schwerlich an den mittelamerika-

3 Vgl. Richter, Sandra: Eine Weltgeschichte der deutschsprachigen Literatur. 2., verbesserte Auflage. München: Bertelsmann Verlag - Random House 2017.

4 Vgl. etwa Losada, Alejandro: La Literatura en la Sociedad de América Latina. Editado por Daniel Cano, Hans-Albert Steger, Roberto Ventura y Ulrich Fleischmann. München: W. Fink Verlag 1987. 5 Vgl. Dessau, Adalbert: Das Internationale, das Kontinentale und das Nationale in der lateinamerikanischen Literatur des 20. Jahrhunderts. In: Lateinamerika (Rostock) (Frühjahrssemester 1978), S. 43-87. 
nischen Raum anschließen und behält ihre eigene Dynamik, so dass wir an dieser Stelle das Modell mit Hilfe der damaligen Forschungen der Chilenin Ana Pizarro erweitern müssen. ${ }^{6}$ Ich werde versuchen, in dieser Vorlesung über das 19. Jahrhundert zwischen zwei Welten die unterschiedlichen Teilregionen auf der amerikanischen Seite entsprechend durch literarische Werke zu repräsentieren und auf diese Weise verschiedenartige kulturelle Areas zu Wort kommen zu lassen.

Ich möchte dabei diese Einteilungen niemals absolut setzen, sondern vielfältige Übergänge berücksichtigen. Denn Schwierigkeiten wird es mit einer allzu schematisch verstandenen Gliederung und Einteilung stets geben. Immerhin aber scheinen mir nicht allein die Grenzen von Nationalem, Regionalem und Kontinentalem oder die Unterscheidung zwischen unterschiedlichen Teilregionen, sondern weit mehr noch die vielfältigen Relationen zwischen unterschiedlichen Areas auf beiden Seiten des Atlantiks auf das hinzudeuten, was die Problematik unseres Gegenstandes ganz wesentlich ausmacht. Allein aus der Perspektive der TransArea Studies lässt sich jene kulturelle Komplexität adäquat beschreiben, welche die Literaturbeziehungen im Jahrhundert der Romantik charakterisiert. $^{7}$

Die methodologische wie didaktische Folgerung für die Anlage unserer Vorlesung sollte daher lauten, auf beiden Seiten des Atlantiks innerhalb des Feldes der romanischen Literaturen der Welt möglichst viele regionale Schwerpunkte und Entwicklungen in unsere Untersuchung einzubauen. Die Repräsentativität bestimmter Areas in Lateinamerika wird daher im Folgenden stets so zugeschnitten sein, dass sie nicht bloß eine regionale kulturelle Verankerung aufweist, sondern Literaturbeziehungen entfaltet, welche in wesentlicher Weise die beiden Welten intertextuell miteinander verbinden und verweben. Ich möchte daher in meiner Darstellung Elemente dessen einfügen, was ich in einer fraktalen Konzeption der Literaturen der Welt entfaltet habe. ${ }^{8}$ Aus jedem literarischen Beispielstext sollen sich daher prinzipiell die Konturen eines WeltFraktals entfalten lassen, anhand dessen gleichsam en miniature ein gesamtes Modell der Literaturen der Welt sichtbar wird. In dieser sehr spezifischen Form scheint es mir möglich zu werden, die Multirelationalität der Literaturen der Welt im 19. Jahrhundert ver-

6 Vgl. u. a. Pizarro, Ana (Hg.): El archipiélago de fronteras externas. Culturas del Caribe hoy. Santiago de Chile: Editorial de la Universidad de Santiago de Chile 2002.

7 Vgl. Ette, Ottmar: TransArea. Eine literarische Globalisierungsgeschichte. Berlin - Boston: Walter de Gruyter 2012.

8 Vgl. Ette, Ottmar: WeltFraktale. Wege durch die Literaturen der Welt. Stuttgart: J.B. Metzler Verlag 2017. 
dichtet und kondensiert, aber dennoch teilweise in einem Close Reading darzustellen. Ich weiß: Das ist die Quadratur des Kreises!

Sie wollen schon einmal wissen, was Sie dann bei einer derartigen Konzeption erwartet? Nun, ich kann Ihnen gerne einige unserer Textbeispiele nennen, anhand derer die unterschiedlichen kulturellen Areas vertreten sein sollen: Da steht José Joaquín Fernández de Lizardis Periquillo Sarniento für Mexiko, Domingo Faustino Sarmientos Facundo für die La-Plata-Regionen des Cono Sur. Cirilo Villaverdes Roman Cecilia Valdés dient als Repräsentant für den karibischen Raum, Clorinda Matto de Turners Aves sin nido für den pazifischen Andenraum sowie für die zirkumkaribische Area der Roman María des Kolumbianers Jorge Isaacs. Aber damit ist nur ein Teil der herangezogenen und auszugsweise einem Close Reading unterzogenen Werke genannt. Denn ich habe noch einige Überraschungen für Sie ...

Somit haben wir bei unserem langen Übergang von der europäischen auf die amerikanische Seite des Atlantiks bereits eine ganze Reihe von Begriffen und Fragestellungen erörtert. Aber eine Problematisierung erlauben Sie mir noch vorab! Denn selbst eine so harmlose und scheinbar faktenbezogene Bezeichnung wie „das 19. Jahrhundert“ hat es in sich. Ist damit der Zeitraum von 1800 bis 1899 gemeint? Das wäre ein Fall für die Statistiker. Wir haben ja bereits im ersten Teil unserer Vorlesung gesehen, dass wir ganz notwendig auf Jean-Jacques Rousseau sowie auf Bernardin de Saint-Pierre - und damit auf Schriftsteller und Philosophen des 18. Jahrhunderts - zurückgreifen mussten, um dieses 19. Jahrhundert adäquat in den Fokus zu rücken.

Fügen wir uns also der Einsicht, dass selbst die zeitlichen Abgrenzungen des von uns behandelten Zeit-Raumes fließend und keineswegs eindeutig sind! Um es mit einem Worte und eindeutig zu sagen: Ich verstehe - literarisch beziehungsweise literarhistorisch gesprochen - unter dem 19. Jahrhundert einen Zeitraum, der in etwa von den 80er Jahren des 18. Jahrhunderts teilweise bis zum Beginn des zweiten Jahrzehnts des 20. Jahrhunderts reicht. Wir haben ja bereits gesehen, welch enorme Bedeutung außereuropäischen Schauplätzen gerade zu Beginn dieses Zeitraumes in den europäischen Literaturen zukommt. Dieses Faktum hat zweifellos mit der Tatsache zu tun, dass zu jener Zeit noch die zweite Phase beschleunigter Globalisierung virulent war und mit dazu beitrug, dass sich die europäischen Literaturen mit ihren Schauplätzen ganz selbstverständlich auf die ganze Welt hin ausdehnten. Betrachten wir nun das Ende unserer skizzierten Zeitspanne, so stellen wir fest, dass sich ab dem letzten Drittel des 19. Jahrhunderts die dritte Phase beschleunigter Globalisierung ansiedelt. ${ }^{9}$ So bilden diese beiden

$9 \mathrm{Zu}$ den vier bisherigen Phasen beschleunigter Globalisierung vgl. Ette, Ottmar: TransArea. Eine literarische Globalisierungsgeschichte. Berlin - Boston: Walter de Gruyter 2012. 
Globalisierungsphasen gleichsam den Rahmen des in dieser Vorlesung behandelten Zeitraums und liefern einen weiteren Grund dafür, unser Zeitfenster in dieser Art zu gestalten.

Das 19. Jahrhundert ist nach meinem Dafürhalten also ein langes Jahrhundert, während man etwa das Jahrhundert der Aufklärung als ein kurzes Jahrhundert begreifen könnte. Denn das „Siècle des Lumières“ könnte man mit dem Tod von Louis XIV. beginnen und bis zum Ausbruch der Französischen Revolution andauern lassen, wodurch dieses philosophische Jahrhundert gerade einmal vierundsiebzig Jahre umfassen würde. Ja, nur für die Statistiker unter uns ist ein so simpler Begriff wie ein Jahrhundert literaturgeschichtlich unproblematisch!

Die romanischen Literaturen der Welt sind in einem 19. Jahrhundert, dessen zwei ,Seiten' wir untersuchen wollen, damit ein ganz bewusstes ästhetisches, philosophisches, literarisches, ideengeschichtliches und selbstverständlich auch literarhistorisches Konstrukt, dem wir uns ebenso sorgsam wie aufmerksam zuwenden wollen. Dass die Pluralität der Literaturen nicht nur für die europäische, sondern auch für die amerikanische Seite gilt, haben wir zur Genüge betont. Doch sprechen wir ebenso von einem Europa, wie wir uns auf ein Lateinamerika beziehen. Dass wir dies tun, hat mit politischen und vor allem kulturellen, nicht zuletzt auch literarischen Gründen zu tun, deren Bedingungen und Kontexte wir uns in dieser Vorlesung ebenfalls erarbeiten werden.

Die oft gestellte Frage, ob es überhaupt ein Lateinamerika gibt, dürfen wir also getrost affirmativ beantworten - und nicht allein mit Blick auf Klausuren, Hausarbeiten, Masterarbeiten oder Doktorarbeiten, in denen von Lateinamerika die Rede ist. Über den Stoff von Examina und Prüfungen sowie über die Existenz großer Forschungseinrichtungen und Institutionen hinaus, die sich der Analyse Lateinamerikas widmen, gibt es diese Welt, weil sich ganze Massen von Menschen als Lateinamerikaner verstehen und als solche ihr je eigenes kulturelles Leben leben. Dies ist eine Realität, und doch zugleich ein Konstrukt: Lateinamerika gibt es nicht von Beginn der Welt an, es handelt sich um ein diskursives Konstrukt, das im Verlauf des 19. Jahrhunderts entstand oder besser: erfunden wurde.

Doch längst nicht alle Bewohnerinnen und Bewohner Lateinamerikas verstehen sich als Lateinamerikaner - auch dies werden wir im Verlauf dieser Vorlesung sehen. Ist ein Amazonasindianer Lateinamerikaner und versteht er sich als solcher? Und was hat er mit einem „ñáñigo“ aus Kuba, einem Maya aus Yukatan oder einem Immobilienspekulanten aus Caracas zu tun? Sind die „Coolie“-Frauen in der Karibik oder die „Curanderas“ in Mexiko ebenso Lateinamerikanerinnen wie die Bewohnerin des einunddreißigsten Stockwerks eines Hochhauses am Strand von Copacabana oder die Salsa-Tänzerin in der Dominikanischen Republik? Wir müssen vorsichtig sein mit Essentialismen, auch wenn es längst üblich ist, von „música latina“ zu sprechen und dabei alles in derselben „Salsa“, in der- 
selben Soße also, zu ersticken. Wir sollten im Verlauf der folgenden Sitzungen uns stets daran erinnern, dass Lateinamerika und Hispanoamerika Konstrukte bleiben - wie ja auch die Realität als ein soziales Konstrukt verstanden werden kann, dessen ,Existenz، oft innerhalb kürzester Zeitspannen als überholt und obsolet gilt und dessen Existenzgrundlagen über Nacht nicht mehr gegeben sind. Denn zu einem bestimmten Zeitpunkt hatte der Begriff „Neuspanien“ ausgedient und war nur noch historisch verfügbar. Warum sollte dies mit dem Begriff „Lateinamerika“ anders sein?

Unsere Vorlesung wird in diesem Teil versuchen, eine Verschiedenheit der Kulturen und verschiedenartigen Identitätszuschreibungen, der unterschiedlichen Ausdrucksformen und populären oder elitären Seins-Weisen, die nicht zum Verschwinden gebracht werden sollen, herauszuarbeiten und in ihrem jeweiligen Wechselspiel mit den europäischen Kulturen und Literaturen zu beleuchten. Denn Differenzen sind das Kapital der Literaturen der Welt; sie sind genau das, was wir in einer Vorlesung, in der wir in verschiedenen Logiken zugleich denken lernen sollen, in den Mittelpunkt rücken müssen und rücken werden.

Wenden wir uns nun aber der faszinierenden Geschichte der literarischen Beziehungen zwischen den iberischen Kolonien in Amerika und Europa zu! Die bekannte US-amerikanische Lateinamerikanistin Jean Franco hat in ihrer noch immer lesenswerten, wenn auch heute teilweise überholten Historia de la literatura hispanoamericana - a partir de la Independencia ${ }^{10}$ der eigentlichen Geschichte seit der Unabhängigkeit ein einleitendes Kapitel vorangestellt, das schlicht mit der Überschrift „La imaginación colonizada“, die kolonisierte Imagination oder Einbildungskraft also, versehen ist. Der Ausdruck ist plakativ und sicherlich nicht schlecht gewählt, doch greift er ein wenig zu kurz. Ist also das Denken, die Phantasie, die kulturelle Ausdruckskraft im kolonialspanischen Lateinamerika nichts weiter als ein kolonisiertes Bewusstsein, ein von außen geprägtes, bestimmtes, man könnte auch sagen ,entfremdetes' Imaginieren?

Die Antwort fällt mir leicht: Dem ist nicht so! Zweifellos ist die Kolonisierung durch äußere Mächte ein Faktum, an dem ebenso wenig zu rütteln ist wie an der teilweisen oder vollständigen Zerstörung bestimmter indigener oder schwarzer Kulturen, die von den europäischen Kolonisatoren auf unmenschliche Weise eingeschränkt und verstümmelt wurden. Aber es wäre doch überaus verkürzend und verfälschend - und Jean Franco hat dies auch nicht in einer absoluten Form getan -, zunächst von einer durch und durch kolonisierten kolonialspanischen Literatur bis zur „Independencia“ und dann, nach der erreichten politischen

10 Vgl. Franco, Jean: Historia de la literatura latinoamericana. A partir de la independencia. Barcelona: Ariel 1979. 
Unabhängigkeit, von einer unabhängig gewordenen Imagination, einer frei und autonom gewordenen Kultur zu sprechen.

Bis in die achtziger Jahre des 20. Jahrhunderts, vereinzelt aber auch noch bis zum heutigen Tag, finden Sie derartige Vorstellungen in der Forschungsliteratur. Sie verstellen vollständig den Blick für die aktiven Formen kultureller Aneignung, für den reichen Formenschatz an Phänomenen, die wir vor allem mit Hilfe des Ortiz'schen Konzepts der Transkulturation präziser verstehen können. Sie verdecken die vielfältigen Möglichkeiten der Resemantisierung ,europäischer Elemente im amerikanischen Kontext, die Refunktionalisierung und Rekontextualisierung bestimmter Schreibformen, Stoffe, Gattungen oder Motive in den kolonialen iberischen Literaturen, die in Amerika geschrieben und weiterentwickelt wurden. ${ }^{11}$ Allein das gewaltige Werk eines Guamán Poma de Ayala hat wahre Forschungsbibliotheken gebildet und deutlich gemacht, wie eigenständig und zugleich viellogisch die kulturellen Entwicklungen auch und gerade in Zeiten asymmetrischer Machtbeziehungen waren. ${ }^{12}$

Es wäre ein Leichtes, derartige Veränderungen bereits im Bordbuch des Christoph Kolumbus ausfindig zu machen, ${ }^{13}$ in einem Text also, der lange Zeit als eine Art Gründungstext der lateinamerikanischen Literatur und insbesondere ihrer Wunderbaren Wirklichkeit, ihres „,real maravilloso“ im Sinne Alejo Carpentiers, gelesen wurde. Die zurückliegenden und in der Kolonialforschung überaus ergebnisreichen Jahrzehnte haben uns durch die erneuerten und methodologisch grundlegend veränderten Forschungsbemühungen ein neues Bild der kolonialspanischen kulturellen Ausdrucksformen und ihrer spezifischen Art transkultureller Aneignung und Kreativität vermittelt. Es ist folglich keineswegs so, dass mit der Independencia etwas völlig Neues in den Blick geraten würde und alles Vorherige als Vorgeschichte, als bloße Prähistorie erschiene.

Es ist daher nicht verwunderlich, dass wir heute im Bereich der Literatur sehr wohl die aktiven Aneignungsprozesse anderskultureller Phänomene stärker

11 Vgl. hierzu Borchmeyer, Florian: Die Ordnung des Unbekannten. Von der Erfindung der neuen Welt. Berlin: Matthes \& Seitz 2009; sowie Fritz, Sabine: Hybride andine Stimmen. Die narrative Inszenierung kultureller Erinnerung in kolonialzeitlichen Chroniken der Eroberten. Hildesheim Zürich - New York: Olms 2009.

12 Vgl. aus dieser Forschungslawine etwa Chang-Rodríguez, Raquel: El discurso disidente. Ensayos de literatura colonial peruana. Lima: Pontifícia Universidad Católica del Perú 1991; oder López-Baralt, Mercedes: Guamán Poma. Autor y artista. Lima: Pontifícia Universidad Católica del Perú 1993.

13 Vgl. hierzu das zweite Kapitel „Vom Auftauchen Amerikas zum Verschwinden Europas“ in Ette, Ottmar: Literatur in Bewegung. Raum und Dynamik grenzüberschreitenden Schreibens in Europa und Amerika. Weilerswist: Velbrück Wissenschaft 2001, S. 85-117. 
betonen müssen und ohne weiteres von einem Polylog zwischen Europa und seinen Kolonien in Amerika sprechen dürfen, der unverkennbar transkulturelle Züge aufweist. Damit soll nicht das Mörderische, das Menschen und Kultur Verachtende bei der sogenannten ,Entdeckung، wie bei der sogleich einsetzenden Eroberung, der „Conquista“ überspielt werden, wie es beispielsweise noch immer im Rahmen der Fünfhundert-Jahr-Feiern des Jahres 1992 geschah, die ja bekanntlich vom „Encuentro de las culturas“, also verharmlosend von der „Begegnung der Kulturen“ sprachen. Es gab sicherlich diese ,Begegnung“, doch ging es - wie man in Mexiko ironisch als Reaktion auf diese Terminologie rasch hervorhob eher um einen „Encontronazo“, einen „Zusammenstoß“ der Kulturen. Dieser löste eher eine Art Trauma aus, das von manchen als Geburtstrauma Lateinamerikas verstanden werden konnte und sich - wie wir sehen werden - im 19. Jahrhundert immer wieder textuell verselbständigte beziehungsweise durch die Textoberfläche brach. Ich meine dennoch, dass man von einem Polylog, einem Gespräch zwischen vielen Stimmen, sprechen darf, solange man sich der Tatsache bewusst bleibt, dass man damit ebenso das Furchtbare wie das Fruchtbare der Ereignisse rund um „Descubrimiento“ und „Conquista“ meint. Der Begriff der Transkulturation eignet sich hierfür weit besser als jener der Alterität ${ }^{14}$ und blendet keineswegs die scharfen Asymmetrien aus, welche die transatlantischen Beziehungen weitestgehend beherrschten. Er macht freilich deutlich, dass es sich eben nicht nur um Prozesse einer Entwertung ganzer Kulturen, einer „Deculturación“ etwa indigener oder schwarzer Kulturen im Sinne von Fernando Ortiz handelte.

Ich möchte daher aus der Perspektive eines derartigen Polylogs zwischen Europa und den Literaturen Amerikas eine Art Grundmuster entwerfen, auf dessen Hintergrund wir die postkolonialen Literaturen des 19. Jahrhunderts im entstehenden Lateinamerika besser einordnen und verstehen können. Denn die Asymmetrie der Beziehungen bestand seit Beginn der sogenannten ,Entdeckung‘, zog sich über die gesamte Kolonialzeit hin, veränderte ihre Stoßrichtung im Jahrhundert der Independencia, aber verschwand auch im 20. Jahrhundert wie in unseren Tagen keineswegs. ${ }^{15}$

Lateinamerikas Literaten der zweiten Hälfte des 20. Jahrhunderts haben es geschafft: Die Namen der Argentinier Jorge Luis Borges und Julio Cortázar, der

14 Vgl. dennoch die in ihrer Zeit sicherlich bahnbrechende Studie von Todorov, Tzvetan: La conquête de l'Amérique. La question de l'autre. Paris: Seuil 1982.

15 Vgl. hierzu Ette, Ottmar: Asymmetrie der Beziehungen. Zehn Thesen zum Dialog der Literaturen Lateinamerikas und Europas. In: Scharlau, Birgit (Hg.): Lateinamerika denken. Kulturtheoretische Grenzgänge zwischen Moderne und Postmoderne. Tübingen: Gunter Narr Verlag 1994, S. 297-326. 
Mexikaner Carlos Fuentes und Octavio Paz, des Peruaners Mario Vargas Llosa, des Kolumbianers Gabriel García Márquez, des Kubaners Alejo Carpentier oder der Chilenin Isabel Allende sind heute einem weltweiten Lesepublikum bekannt. Werke dieser Autorinnen und Autoren haben längst auch im deutschsprachigen Raum eine treue Gemeinde von Leserinnen und Lesern gefunden und die literarische Kreativität des Subkontinents in den vergangenen Jahrzehnten buchstäblich vor Augen geführt. Daneben hat die Fülle an Filmen, Publikationen und Kommentaren zu den Fünfhundert-Jahr-Feiern anlässlich der sogenannten ,Entdeckung‘ Amerikas den Blick gerade der Europäer ab 1992 auf einen ganz bestimmten Zeitraum gelenkt. Dieser wurde schon von den herausragenden Kolonialgeschichtsschreibern des ausgehenden 18. und beginnenden 19. Jahrhunderts - Guillaume-Thomas Raynal, William Robertson und Alexander von Humboldt - in seltener Einmütigkeit als der wichtigste Augenblick innerhalb der weltgeschichtlichen Entwicklung der Neuzeit bezeichnet. Doch wir sollten uns davor hüten, mit der massiven Durchsetzung lateinamerikanischer Literaturen auf einer weltumspannenden Ebene die endgültige Etablierung symmetrischer Literaturbeziehungen im transatlantischen Kontext gleichzusetzen.

An dieser Stelle müssen wir präziser analysieren und dürfen nicht die Augen davor verschließen, dass sich die historisch akkumulierten Asymmetrien keineswegs aufgelöst, sondern im 20. und beginnenden 21. Jahrhundert nur variantenreich umgestaltet haben. Denn ebenso, wie die großen Erfolge vor allem der zweiten Hälfte des 20. Jahrhunderts die literarische Vorgeschichte teilweise stark verdunkelten, erweckte auch die vorrangige Beschäftigung mit der Figur des Christoph Kolumbus sowie der Chronisten der ersten Stunde oftmals den Eindruck, als habe die Literatur erst mit dem Genuesen Einzug in Amerika gehalten. Gerade so, als habe es nur eine einzige, eine schriftkulturelle Tradition in der von den Europäern so benannten ,Neuen Welt' gegeben. Doch oftmals ist das genaue Gegenteil der Fall. Was also wissen wir wirklich vom Schreiben in Lateinamerika? Warum hat nur eine bestimmte Literatur zu uns gefunden? Und wie hängt dies mit unserer Lesart lateinamerikanischer Texte zusammen?

Halten wir an dieser Stelle fest: Die ganze kulturelle Vielfalt und multirelationale Vielgestaltigkeit Lateinamerikas, die gerade auch für den Bereich der Literaturen dieses Raumes charakteristisch ist, wurde in Europa von einem breiten Publikum erst in Ansätzen wahrgenommen! Mehr noch: was zwischen Kolumbus’ Bordbuch und den Fiktionen von Jorge Luis Borges oder García Márquez' Hundert Jahre Einsamkeit geschrieben wurde, ist hierzulande nur den universitären Spezialisten bekannt und kaum in deutscher Sprache zugänglich. Gerade das 19. Jahrhundert der Literaturen Lateinamerikas ist in Europa, aber auch in einem weltweiten Maßstab weitestgehend unbekannt. 
Während die Übersetzungen großer Romane bekannter Autorinnen und Autoren des 20. und beginnenden 21. Jahrhunderts auch für deutschsprachige Verlage lukrative Perspektiven bieten, wagt sich kaum einmal ein Verleger an Texte aus dieser vergessenen ,Zwischenzeit' heran. Im Bereich literarischer Entwicklungen erschien Lateinamerika - unter welchen Vorzeichen auch immer - von Europa aus gesehen wie ein einziger homogener Block, die ,großen“ lateinamerikanischen Autor`innen als Repräsentant ${ }^{\star}$ innen einer einzigen lateinamerikanischen Literatur. Die Ausschnitthaftigkeit eines solchen Bildes der lateinamerikanischen Literatur ist längst zu einer kulturellen Selbstverständlichkeit geworden, welche bei den entscheidenden Vermarktungsstrategien lateinamerikanischen Schreibens nicht einmal mehr mitgedacht und reflektiert werden muss. ${ }^{16}$ Daher ist es unumgänglich, die Entstehungsbedingungen und historischen Hintergründe des Schreibens in dem aufzuarbeiten, was sich ab der Mitte des 19. Jahrhunderts zunehmend als Lateinamerika bezeichnete, um die Literaturen dieser kulturellen Areas in einen gleichrangigen Polylog auf Augenhöhe mit jenen Europas miteinzubeziehen.

Ein derartiger Polylog kann freilich nur in Gang kommen, wenn man bereit ist, sich auf die spezifische Andersartigkeit lateinamerikanischer Literaturen in ihren vielfältigen Beziehungen zu den Literaturen Europas einzulassen. Dabei geht es mir in diesem Zusammenhang nicht vorrangig um den Status einer Alterität, einer Andersartigkeit der Literaturen Lateinamerikas, als vielmehr um eine Weitung und Erweiterung von Denkmöglichkeiten und Logiken, in welche die Literaturen Lateinamerikas miteinzubeziehen sind. ${ }^{17}$ Dies impliziert gleichsam nebenbei eine fundamentale Erhöhung an Vorstellungsmöglichkeiten, für welche die Literaturen der Welt ohnehin maßgeblich sind.

Unabdingbare Voraussetzung für eine Überwindung asymmetrischer Beziehungen ist ein Verständnis der historischen Entwicklungslinien, welche die Herausbildung dieser Literaturen charakterisierten. Dabei kann man zunächst getrost davon ausgehen, dass die kolonialen Literaturen der sogenannten ,Neuen Welt' bis zum Ende des 19. Jahrhunderts - genauer: bis zum hispanoamerikanischen Modernismo - für die europäischen Literaturen von vernachlässigbarer Bedeutung waren. Genau wie wir dies am Beispiel der haitianischen „littérature jaune“ sahen, mochte man ,diesen Literaturen“ ja ein eifriges Bemühen einräu-

16 Vgl. hierzu kritisch Müller, Gesine: Wie wird Weltliteratur gemacht? Globale Zirkulationen lateinamerikanischer Literaturen. Berlin - Boston: Walter de Gruyter 2020.

17 Vgl. Ette, Ottmar: Ottmar: Weiter denken. Viellogisches denken / viellogisches Denken und die Wege zu einer Epistemologie der Erweiterung. In: Romanistische Zeitschrift für Literaturgeschichte / Cahiers d'Histoire des Littératures Romanes (Heidelberg) XL, 1-4 (2016), S. 331-355. 
men, doch erblickte man in ihnen bestenfalls hundertprozentige Nachahmungen, die sich an europäischen Vorbildern ausrichteten, hielt man die Autor ${ }^{\star}$ innen aus marginalen Weltregionen doch bis ins 20. Jahrhundert hinein nicht für fähig, eigenständig kreativ zu sein und Neues hervorzubringen. Wir werden im weiteren Verlauf der Vorlesung sehen, dass dies nichts war als ein weiteres rassistisches Vorurteil, das man in Europa hinsichtlich der Literaturen der außereuropäischen Welt sorgsam hegte und pflegte.

Dies sollte uns freilich nicht dazu verleiten, einen historischen Überblick erst mit dem Modernismo im Fin de siècle des 19. Jahrhunderts beginnen zu lassen. Wir werden uns diesen Literaturen des „Fin de siglo“ mit gebührender Aufmerksamkeit zuwenden und uns dabei mit Autorinnen und Autoren beschäftigen, welche die europäischen Vorstellungen eines finisekulären Schreibens buchstäblich sprengten. Doch zu fragen ist vielmehr, was die besondere Bedeutung des gesamten 19. Jahrhunderts für die literarischen Beziehungen zwischen Lateinamerika und Europa ausmacht.

Denn es ist selbstverständlich keineswegs so, dass es zuvor im geistigen und literarischen Bereich keine Austauschbeziehungen zwischen Europa und Lateinamerika gegeben hätte. Ganz im Gegenteil: Der kubanische Essayist und Kulturkritiker Roberto Fernández Retamar hat dies einmal auf die Formel gebracht, das Gold des „Siglo de Oro“, des Goldenen Zeitalters der spanischen Literatur, stamme aus Amerika. Er hatte dabei allerdings nicht die lateinamerikanischen Literaturen, wohl aber einen bestimmten Teil der asymmetrischen wirtschaftlichen Beziehungen zwischen Spanien und dessen Kolonien im Sinn. Fernández Retamar hat mit guten Gründen darauf verzichtet, eine direkte literarische Beziehung zu konstruieren. Dabei konnte Amerika sehr wohl Thema europäischen Schreibens sein - nicht zuletzt gerade bezüglich jener ökonomischen Asymmetrien, die auch innerhalb Europas selbst beobachtbar waren. Quevedos berühmte Klage, dass das Geld, „Don Dinero“, in Amerika „ehrenhaft“ das Licht der Welt erblicke, dann aber „zum Sterben nach Spanien“ käme und schließlich „,in Genua begraben liege“, lässt sich als in Verse gefasste Kritik eines Spaniers an der wirtschaftlichen Abhängigkeit seines Landes innerhalb Europas lesen, nicht aber als Kritik am transatlantischen Kolonialsystem selbst. Es gibt im Übrigen eine sehr gelungene Vertonung dieses Gedichts von Francisco de Quevedo durch Paco Ibáñez, welche zwar nicht alle Strophen enthält, die Sie sich aber leicht über das Internet besorgen können. Da es sehr schöne und witzige Übersetzungen ins Französische und ins Englische gibt, will ich Ihnen diese gerne beifügen: 
Madre, yo al oro me humillo él es mi amante y mi amado pues de puro enamorado de continuo anda amarillo; que, pues, doblón o sencillo, hace todo cuanto quiero, poderoso caballero es Don Dinero

Nace en las Indias honrado, donde el mundo le acompaña: viene a morir en España y es en Génova enterrado; $y$, pues quien le trae al lado es hermoso, aunque sea fiero, poderoso caballero

es Don Dinero.

Es galán, y es como un oro, tiene quebrado el color; persona de gran valor, tan cristiano como moro; pues que da y quita el decoro y quebranta cualquier fuero, poderoso caballero es Don Dinero.

Son sus padres principales, y es de nobles descendiente, porque en las venas de Oriente todas las sangres son reales; $\mathrm{y}$, pues quien hace iguales al duque y al ganadero, poderoso caballero es Don Dinero. Mas, ¿a quién no maravilla ver, en su gloria sin tasa, que es lo menos en su casa doña Blanca de Castilla? Pero, pues da al bajo silla $\mathrm{y}$ al cobarde hace guerrero, poderoso caballero es Don Dinero.

Sus escudos de armas nobles son siempre tan principales, que sin sus escudos reales no hay escudos de armas dobles, $\mathrm{y}$, pues a los mismos nobles da codicia su mimero, poderoso caballero
Mère, moi je m'humille à l'or, Il est mon amoureux et aimé, Puisque de pur amoureux, Il est constamment jaune. Puisque doublon ou petite monnaie, Il fait tout ce que je veux, Puissant galant

Et Monsieur Argent.

Il naît honnête aux Indes, Où le monde l'accompagne; Il vient à mourir en Espagne, Et est enterré à Gênes.

Puisque pour qui l'amène à coté

Il est beau, même si féroce, Puissant galant

Est Monsieur Argent.

Il est galant comme une médaille d'or, Il est de couleur inégale, Une personne de grande valeur, Comme Chrétien et Maure.

Puisqu'il donne et coupe le protocole Et casse n'importe quel pouvoir, Puissant galant

Est Monsieur Argent.

Ses parents sont des chefs, Et il est issu des nobles, Parce que dans les veines d'Orient Tous les sangs sont royaux. Puisqu'il rend égaux Riche et Mendiant, Puissant galant

Est Monsieur Argent.

Qu'il n'est pas émerveillé

De voir aux anges, sans taxation, Qui est le plus vil de sa maison,

A Madame Blanche de Castille?

Puisque sa force humille

Le lâche et le brave,

Puissant galant

Est Monsieur Argent [...].

On his shields are noble bearings;

His amblazonments unfurling

Show his arms of royal sterling

All his high pretensions airing;

And the credit of his miner

Stands behind the proud refiner, Over kings and priests and scholars 
es Don Dinero.

Por importar en los tratos

y dar tan buenos consejos, en las casas de los viejos gatos le guardan de gatos; y, pue, él rompe recatos y ablanda al juez más severo, poderoso caballero es Don Dinero.

Y es tanta su majestad, aunque sus duelos son hartos, que con haberle hecho cuartos, no pierde su autoridad; pero, pues da calidad al noble y al pordiosero, poderoso caballero es Don Dinero. Nunca vi damas ingratas a su gusto y aficción, que a las caras de un doblón hacen sus caras baratas; $\mathrm{y}$, pues hace las bravatas desde una bolsa de cuero, poderoso caballero es Don Dinero.

Más valen en cualquier tierra -¡mirad si es harto sagaz!sus escudos en la paz que rodelas en la guerra; $\mathrm{y}$, pues al pobre le entierra y hace propio al forastero, poderoso caballero es Don Dinero.
Rules the mighty Lord of Dollars.

Contacts, bonds, and bills to render, Like his counsels most excelling, Are esteemed with the dwelling Of the banker and the leader. So is prudence overthrown, And the judge complaisant grown,Over kings and priests and scholars Rules the mighty Lord of Dollars. Such indeed his sovereign standing (With some discount in the order), Spite the tax, the cash-recorder Still his value fixed is branding. He keeps rank significant To the prince of finn in want,Over kings and priests and scholars Rules the mighty Lord of Dollars. Never meets he dames ungracious To his smiles or his attention, How they glow but at the mention Of his promises capacious! And how bare-faced they become To the coin beneath his thumb Over kings and priests and scholars Rules the mighty Lord of Dollars. Mightier in peaceful season (And in this his wisdom showeth) Are his standards, than when bloweth War his haughty blasts and breeze on; In all foreign lands at home, Equal e'en in pauper's loam,Over kings and priests and scholars Rules the mighty Lord of Dollars. ${ }^{1}$

1 Quevedo, Francisco: Poesías de D. Francisco Gomez de Quevedo y Villegas. Madrid: Libro de oro 1859, S. 59 ff. Französische Übersetzung von Alvaro Vigueras Vilchez, Englisch von Thomas Walsh. Online unter https://lyricstranslate.com/de/poderoso-caballero-es-don-dinero-puissantgalant-est-monsieur-argent.html, zuletzt aufgerufen am 12.11.2020. 


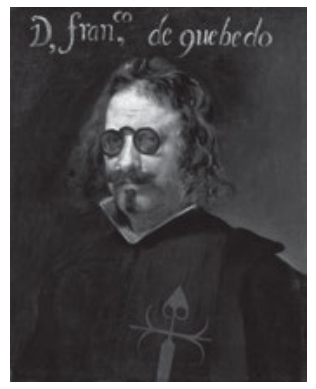

Abb. 27: Francisco de Quevedo (Madrid, 1580 - Villanueva de los Infantes, Provinz Ciudad Real, 1645).

Spätestens beim Lesen dieser Verse wird uns zum einen deutlich, dass wir es seit der ersten Phase beschleunigter Globalisierung mit der Herausbildung eines kapitalistischen Handels- und Produktionssystems im Weltmaßstab zu tun haben. Diese Entwicklung begann folglich mit dem Ausgreifen der iberischen Mächte mit Unterstützung italienischer und später auch deutscher Banken nach Amerika angelehnt an das Bankhaus der Fugger wird übrigens noch heute ein reicher Mann in Spanien als „fúcar“ bezeichnet. Zum zweiten ist dieses kapitalistische System bereits ganz an der Abschöpfung von Kapital und anderen Reichtümern in den Kolonien ausgerichtet; ein in einem zuvor unbekannten Maßstab akkumuliertes Kapital, welches dann in Europa für Kriege oder den Erwerb von Luxusgütern eingesetzt werden konnte. Denn ohne die satten Erträge aus den Kolonien wären die großen finanziellen Spielräume nicht nur in den kolonialen Mutterländern, sondern auch den mit diesen wirtschaftlich und finanziell verflochtenen europäischen Staaten und damit der gesamte Aufstieg Europas seit der ersten Phase beschleunigter Globalisierung nicht wirklich nachvollziehbar.

Francisco de Quevedos Gedicht führt uns überdies und gleichsam nebenbei ein in die Thematik dessen, der ständig auf der Suche nach Geld ist: Es ist die Geschichte des (spanischen) „Pícaro“, ein Thema, das - wie wir bald sehen werden - auch auf dem amerikanischen Kontinent aufgenommen und in ein literarisches Meisterwerk umgeformt wurde. Unübersehbar ist, dass der von Quevedo im Gedicht angesprochene Verfall traditioneller Werte und sozialer Hierarchien gerade auch in den spanischen Besitzungen der ,Neuen Welt' gesellschaftspolitisch virulent wurde und eine enorme Sprengkraft am Vorabend der Independencia, der politischen Unabhängigkeit von Spanien, entfalten konnte. Der „Pícaro“, der Schelm im Roman, wurde zu einer Gestalt der lateinamerikanischen Prosa, die bis heute wenig von ihrer Faszinationskraft verloren hat.

Doch kehren wir zu unserer bei Quevedo angerissenen Thematik zurück! Noch viel weniger wäre es für den spanischen Dichter und seine Zeitgenossen denkbar gewesen, dass neben dem Geldsegen aus den überseeischen Kolonien auch literarische Formen und Traditionen aus Amerika in Spanien eine breite 
Aufnahme hätten finden können. Die kulturellen Selbstverständlichkeiten im Europa jener Epoche schoben derlei Gedanken und Praktiken einen Riegel vor. Im günstigsten Falle erweckten die kulturellen Erzeugnisse aus der Neuen Welt Bewunderung gemischt mit Verwunderung, ohne dass aber eigene kreative Ausdrucksmöglichkeiten neue Impulse erhalten hätten.

So notierte kein Geringerer als Albrecht Dürer im Jahre 1520 in seinem Tagebuch der Niederländischen Reise, nachdem er in Brüssel die von Hernan Cortés an die spanische Krone übersandten und dort ausgestellten aztekischen Schätze gesehen hatte:

Diese ding sind alle köstlich gewesen, das man sie beschäczt umb hundert tausent gulden werth. Und ich hab aber all mein lebtag nichts gesehen, das mein hercz also erfreuet hat als diese ding. Denn ich hab darin gesehen wunderliche künstliche ding und hab mich verwundert der subtilen ingenia der menschen in frembden landen. Und der ding weiß ich nit außzusprechen, die ich do gehabt hab. ${ }^{18}$

Das Fremde erscheint in dieser Passage als das Wunderliche, Wunderbare, das Verwunderung hervorrufen kann, das jedoch keinen Eingang in das Eigene finden wird. Dürers künstlerische Offenheit ist gleichwohl beeindruckend, spricht er doch von der subtilen Ingeniosität der von ihm gesehenen Kunstwerke. Das Thema der Wunderbaren Wirklichkeit, des „real maravilloso“, wie es Alejo Carpentier entwickeln sollte, deutet sich hier aus europäischer Perspektive bereits an und entfaltet seine ganze Macht weit über die historischen Grenzen der Kolonialzeit hinaus.

Eine wirkliche kreative Auseinandersetzung mit diesem Wunderbaren aus europäischer Perspektive findet jedoch nicht statt. Denn die Alterität, die Andersartigkeit des Gesehenen, erscheint als zu groß, als dass sie kurzfristig zu überbrücken gewesen wäre. Dies gilt in vielleicht noch stärkerem Maße für den literarischen Bereich und textuelle Ausdrucksformen. Über punktuelle Kulturberührungen ${ }^{19}$ hinaus, die sich aus der konkreten Lebensgeschichte einzelner Autoren ergeben, lassen sich keine Indizien für eine wie auch immer geartete ,Beeinflussung“ europäischer Schriftsteller durch die Literatur feststellen, die in den spanischen Kolonien geschrieben wurde.

18 Dürer, Albrecht: Das Tagebuch der Niederländischen Reise. 1520-1521. Herausgegeben von J.-A. Goris und G. Marlier. Brüssel 1970, S. 65; die Passage wird kommentiert in Gewecke, Frauke: Wie die neue Welt in die alte kam. Stuttgart: Klett-Cotta 1986, S. $150 \mathrm{f}$.

19 Vgl. zur Terminologie von Kulturberührung, Kulturkontakt usw. das Werk von Bitterli, Urs: Die „Wilden“ und die „Zivilisierten“. Die europäisch-überseeische Begegnung. München: Deutscher Taschenbuch Verlag 1982. 
Für die nachfolgenden Überlegungen wird es von großer Bedeutung sein, im literarischen wie nicht-literarischen Bereich Richtungen und Bedingungen des Informationsflusses zu untersuchen, der sich zwischen Europa und Lateinamerika, später dann auch zwischen dem Norden und dem Süden des amerikanischen Doppelkontinents entwickeln sollte. Dabei werden unterschiedliche Asymmetrien dieses Flusses an Informationen ohne Zweifel zu beobachten sein. Gerade aus einer solchen Perspektive soll die besondere Bedeutung jener Beziehungen erkennbar werden, welche die modernistischen Autoren Hispanoamerikas zu den literarischen oder philosophischen Werken Europas herstellten und sich damit erstmals eine bewusst eigenständige Position erarbeiteten, welche das 20. Jahrhundert und dessen spezifische Entwicklungen vorbereitete. In diesem 20. Jahrhundert kamen wesentliche Impulse für die ,westlichen' Literaturen Europas und der USA wohlgemerkt aus Lateinamerika. In unserer Vorlesung über das 20. Jahrhundert haben wir nicht umsonst festgestellt, dass Autoren wie etwa der Argentinier Jorge Luis Borges von grundlegender Bedeutung für die Entfaltung eines weltweiten Schreibens im Zeichen der Postmoderne wurden.

Seit Beginn der Eroberung Amerikas vor weit mehr als fünfhundert Jahren wurde die ,Neue Welt‘ ebenso partiell, nach einzelnen Areas unterschieden, wie sukzessiv in einen wirtschaftlichen, sozialen und politischen Prozess integriert, der fremdgesteuert von Spanien und Portugal aus seine Impulse erhielt. Die kolonialen Ergänzungs- und Ausplünderungsgebiete wurden dabei in zunehmend enger verflochtene Abhängigkeitsbeziehungen mit den sogenannten ,Mutterländern‘ eingebaut. Die Beschleunigung einer Vielzahl politischer, sozialer oder wirtschaftlicher Entwicklungen durch den kolonialen Ausgriff der iberischen Mächte auf Amerika in diesen Mutterländern selbst steht heute außer Frage. Denn die Finanz-, Wirtschafts- oder Sozialbeziehungen der iberischen Mächte wurden durch die von ihnen ausgehenden Kolonialstrukturen grundlegend geprägt. Die Etablierung eines weltweiten kapitalistischen Systems beruhte von Beginn an auf der Ungleichverteilung von Macht und Gewalt sowie auf Ausplünderung ,peripherer‘ Kolonialgebiete.

Dabei vollzog sich die Umwandlung der Kolonien in zu Europa komplementäre Wirtschaftsgebilde über die städtischen Zentren der neu geschaffenen staatlichen Strukturen. Nicht nur im politischen oder ökonomischen, sondern auch im kulturellen Bereich wurden auf diese Weise Beziehungen aufgebaut, die beide Kontinente - Europa wie Amerika - in einen gemeinsamen, aber gegensätzlichen und äußerst widerspruchsvollen Raum verwandelten, der als ein Bewegungsraum bewegungs- und nicht raumgeschichtlich aufzufassen ist.

Die Kolonialstädte waren dabei einzeln an ihre jeweiligen Metropolen angebunden, kaum aber untereinander liiert. So entstand auch auf dem amerikanischen Kontinent eine Archipel-Situation mit deutlicher Zentrierung auf Europa 
und einer klar unterentwickelten internen Relationalität der so gebildeten Inseln untereinander. Diese Tatsache führte den brasilianischen Anthropologen Darcy Ribeiro zu der Frage, ob es Lateinamerika denn überhaupt gebe:

Die geographische Einheit hat in Lateinamerika nie zu einer politischen Einheit geführt, weil die verschiedenen Kolonien, aus denen die lateinamerikanischen Gesellschaften hervorgegangen sind, jahrhundertelang ohne Kontakt nebeneinander bestanden haben. Jede einzelne war indirekt an die Metropole gebunden. Noch heute leben wir Lateinamerikaner wie auf einem Archipel, dessen Inseln miteinander durch Schiffe und Flugzeuge verbunden sind und die mehr nach außen auf die weltwirtschaftlichen Zentren hin ausgerichtet sind als nach innen. Sogar die Grenzen der lateinamerikanischen Länder verlaufen längs der unbewohnten Kordillere oder dem undurchdringlichen Urwald, und sie isolieren mehr, als dass sie verbinden, und sie erlauben selten einen intensiven Kontakt. ${ }^{20}$

In dieser Passage wird nicht mit Blick auf die Karibik, sondern auf ganz Lateinamerika von einer Archipel-Situation gesprochen, mit der wir uns im weiteren Verlauf der Vorlesung noch vielfach beschäftigen werden. Diese Aussage wurde im Übrigen nicht gegen Ende der Kolonialzeit getroffen, sondern Ende der siebziger Jahre des 20. Jahrhunderts. Sie sehen: Die Archipel-Situation, die durch koloniale iberische Strukturen geschaffen oder doch zumindest nicht behindert wurde, ist entgegen aller Vermutungen durch die politische Unabhängigkeit zu Beginn des 19. Jahrhunderts keineswegs überwunden worden; sie dauern vielmehr unter den Bedingungen unabhängiger Nationalstaaten bis heute an.

Aus diesen Gründen ist es stets nützlich, mit Blick auf Lateinamerika zwischen einer internen und einer externen Relationalität zu unterscheiden, Begrifflichkeiten, die in unserer Vorlesung bereits Anwendung gefunden haben und nun ihren methodologisch bestimmten Platz einnehmen. Abhängigkeit und Außenorientierung prägten von Beginn an auch den kulturellen Sektor innerhalb der rasch errichteten asymmetrischen Kolonialstrukturen. Der Kolonial-Stadt kam dabei die Funktion eines Hortes der Schrift zu. Der Aufbau administrativer Strukturen nach europäischem, das heißt schriftkulturellem Vorbild und die Entstehung einer kolonialen Literatur hängen aufs Engste miteinander zusammen. Sie sind weder von der Schrift- oder Buchkultur der christlichen Religion noch von den auf Schrift basierenden Verwaltungsstrukturen zu trennen, welche Portugal und Spanien in ihren Kolonien rasch einführten und mit geeigneten Verwaltungsbeamten untersetzten.

20 Ribeiro, Darcy: Gibt es Lateinamerika? In (ders.): Unterentwicklung, Kultur und Zivilisation. Ungewöhnliche Versuche. Frankfurt am Main: Suhrkamp 1980, s. 315. 
Was aber ist mit dem Begriff „Schrift“ in diesem Zusammenhang gemeint? Die eurozentrische Definition von Schrift, der Ausschluss aller dieser Norm nicht genügenden Schriftsysteme (etwa Piktogramme oder Knotenschriften) und die auf der jüdisch-christlichen Tradition beruhende Gleichsetzung von Buchstabenschrift, historischer Überlieferungsfähigkeit und Wahrheitsanspruch ${ }^{21}$ tilgten das kulturell Andere aus der Vorstellungswelt der kolonialspanischen Eliten, aus dem von ihnen abgesteckten Raum der Kultur. Die indigene Bevölkerung, die ,Wilden“ und ,Nicht-Zivilisierten', konnten in den Augen der Europäer unter keinen Umständen über eine Kultur verfügen - und eben dort, wo man auf die unterschiedlichsten indigenen Aufbewahrungs- und Schriftsysteme stieß, versuchte man, diese alsbald zu vernichten.

Dem so definierten kulturellen Raum der Städte, der „ciudades letradas“, ${ }^{22}$ diente das Nicht-Urbane, dienten die weiten amerikanischen Territorien gleichsam als Möglichkeit kontrastiver Abgrenzung. Der Raum der Kultur bildete sich in Opposition zu einem ihn umgebenden Raum von Wildheit und Barbarei. An diese aus der Kolonialzeit ererbten Strukturen knüpften insbesondere im 19. Jahrhundert Identitätsentwürfe für Lateinamerika an, die dem barbarischen Raum der amerikanischen Natur die Urbanität einer europäischen Zivilisation in Amerika entgegenstellten. So wird der uruguayische Essayist José Enrique Rodó in seinem zum neuen Jahrhundert im Jahr 1900 veröffentlichten Ariel auf den argentinischen Literaten und Politiker Domingo Faustino Sarmiento hinweisen. Dieser hatte in seinem 1845 erstmals veröffentlichten Facundo den so verstandenen Gegensatz zwischen Zivilisation und Barbarei literarisch brillant formuliert und - einmal an die politische Macht gelangt - in eine gerade auch gegenüber den Indianern unversöhnliche Politik umgesetzt. Auch Rodó wird in seinem für ein sich auf seine lateinischen ,Wurzeln“ besinnendes Lateinamerika wegweisenden Entwurf den indigenen Kulturen keinerlei Platz einräumen. ${ }^{23}$

Der Ausschluss von Mündlichkeit und nicht-alphabetischen Schriftsystemen setzt sich noch bis in die heutigen Literaturgeschichten hinein fort; erst seit den achtziger Jahren des 20. Jahrhunderts bahnt sich auf diesem Gebiet ein Paradig-

21 Vgl. Mignolo, Walter D.: La historia de la escritura y la escritura de la historia. In: Forster, Merlín H. / Ortega, Julio (Hg.): De la crónica a la nueva narrativa mexicana. Coloquio sobre literatura mexicana. México: Editorial Oasis 1986, S. 13-28.

22 Vgl. hierzu Rama, Angel: La ciudad letrada. Hanover: Ediciones del Norte 1984; sowie zu diesem Begriff des Uruguayers Rama die programmatische Studie von Adorno, Rolena: La „ciudad letrada“ y los discursos coloniales. Inb: Hispamérica (Gaithersburg) 48 (1987), S. 4-24.

23 Vgl. hierzu Vorwort und Nachwort zu meiner Übersetzung von Rodó, José Enrique: Ariel. Übersetzt, herausgegeben und erläutert von Ottmar Ette. Mainz: Dieterich'sche Verlagsbuchhandlung 1994. 
menwechsel an, der zu einer grundlegenden Erweiterung des Literaturbegriffs in Hinblick auf die Vielfalt der Sprachen, Diskurse und Zeichensysteme während der Kolonialzeit in Amerika führt. In Guatemala etwa ist die kulturelle und politische Gleichstellung des Maya-Quiché noch immer ebenso prekär wie ein Literaturpreis für in indigenen Sprachen abgefasste Texte, den der guatemaltekische Schriftsteller Rodrigo Rey Rosa für sein Heimatland gestiftet hatte. Die ehedem „kolonialspanische Literatur“ könnte so dereinst als nur mehr eine unter vielen diskursiven Praktiken im literarischen Raum der Gegenwart erscheinen. Dass sie es bereits in der Kolonialzeit war, aber die im indigenen Bereich konkurrierenden Praktiken zum Verschwinden zu bringen suchte, ist in der heutigen Forschungsliteratur unstrittig. Die bereits im 19. Jahrhundert vehement in Guatemala vom Kubaner José Martí geforderte Gleichstellung der indigenen Bevölkerung ist auch noch in unserer Gegenwart ein fernes Ziel, das gleichwohl irgendwann in hoffentlich möglichst bald eintretender Zukunft erreicht werden wird.

Die literarischen Beziehungen zwischen Spanien und Portugal, den beiden europäischen Führungsmächten der ersten Phase beschleunigter Globalisierung, und ihren amerikanischen Kolonien sind also von Beginn an ebenso asymmetrisch wie der Wissenstransfer zwischen den beiden ungleichen Kontinenten. Einerseits werden europäische Normen und Vorstellungen im (und über den) kulturellen Bereich in den amerikanischen Kolonien durchgesetzt oder übernommen; andererseits ist der Informationsfluss von Amerika nach Europa an den Informationsbedürfnissen und Durchsetzungsansprüchen der hegemonialen Zentren orientiert. Das ist für uns im 21. Jahrhundert gar nicht so schwer vorstellbar. Denn noch heute können wir ohne weiteres diese Asymmetrie in unserem scheinbar so weltweit verwobenem Gewebe feststellen, dienen doch die Auslands-Korrespondenten europäischer Sender überwiegend dazu, Lateinamerika in den Nachrichten als Subkontinent von Naturkatastrophen, Finanzkrisen oder staatlicher Unterdrückung zu porträtieren und ein kaum dieser komplexen Welt adäquates Nachrichtenbild in den Medien Europas, aber auch der USA entstehen zu lassen.

$\mathrm{Zu}$ Zeiten des iberischen Kolonialismus erfüllten Chronisten wie Missionare eine kaum zu überschätzende Funktion innerhalb des Wissenstransfers und Informationsflusses zwischen Kolonie und Mutterland. In den spanischen Chroniken des 16. Jahrhunderts werden ausgegrenzte kulturelle Praktiken, etwa bestimmte Riten, Vorstellungen oder nicht-schriftliche Überlieferungsmöglichkeiten, angesichts der alles überwachenden Inquisition oft nur mit dem Hinweis darstellbar, dass ein Wissen über derartige (,satanische') Praktiken eine nachfolgende Christianisierung erleichtern könne. Im Wissenstransfer von Europa nach Lateinamerika spielte gerade die den Europäern kulturell selbstverständliche Schriftpraxis eine entscheidende Rolle bei der Missionierung, war doch das Wort Gottes in der Schrift der Bibel, im Buch der Bücher, aufgehoben. Diese Dominanz der Schrift 
und des Schriftlichen in der kolonialen „ciudad letrada“ war von ungeheurer Bedeutung und wirkte auch nach der Independencia fort. Die Schriftstellerinnen und Schriftsteller im sich befreienden Amerika des 19. Jahrhunderts sind noch immer die Erben jener Entwicklung.

Diese Fixierung auf die Buchstabenschrift war jedoch trotz all ihrer zerstörerischen Wirkung - denken wir nur an die gezielte Vernichtung indianischer Kulturgüter und Aufzeichnungsformen durch kolonialspanische oder kirchliche Organe - weit davon entfernt, die kulturelle Vielfalt auf dem amerikanischen Kontinent auszulöschen. Sie führte aber sehr wohl eine Hierarchie ein, die andere kulturelle Praktiken in ausgegrenzte (und diffamierte) soziale Räume abdrängte. Damit leistete sie einer grundlegenden kulturellen Heterogenität und mehr noch Hybridität Vorschub, welche die Kulturen Lateinamerikas bis heute kennzeichnet. $^{24}$

Erkennbar wird dies, wenn wir den vermeintlich absoluten Gegensatz zwischen schriftlichen, das heißt auf einer alphabetischen Schrift beruhenden, und nicht-schriftlichen Tradierungsmöglichkeiten überwinden und die kreative Anverwandlung fremder kultureller Praktiken durch indianische Schriftkundige betrachten. Veranschaulichen wir dies an einem kurzen Beispiel aus dem neuspanisch-nordamerikanischen Raum! Dort wird der Übergang von vorherrschend mündlichen zu vorherrschend schriftlichen Ausdrucksformen in einem Bericht von Domingo Francisco de San Antón Muñón Chimalpahin Quauhtlehuanitzin oder einfach Chimalpahin aus dem 16. Jahrhundert als Kontinuum dargestellt:

\begin{abstract}
Dann wurden das bemalte Papier und die Geschichte der Erblinien seinem geliebten Sohne, dem Herrn Don Domingo Hernández Ayopochtzin, überlassen, der sich in der Wissenschaft des Erzählens der Bücher unterrichtet hatte und ein Buch malte, das er ganz in Buchstaben ohne alle Zusätze schrieb, wie ein getreuer Spiegel der Dinge, die er von dort übertrug. Neuerlich habe ich nun gemalt und habe mit Buchstaben ein Buch geschrieben, in welchem ich alle alten Geschichten vorgelegt habe. ${ }^{25}$
\end{abstract}

Chimalpahin entstammte der alten Adelsschicht der Mexica oder Azteken und schrieb auf Spanisch wie auf Náhuatl, war in den alten Künsten bewandert und hatte alle Techniken der spanischen Eroberer erlernt, was ihm erlaubte, Berichte über die Herrschaft der alten Adelsfamilien und deren Herkunft zu verfassen. Er trat aber auch als sorgfältiger Chronist zeitgeschichtlicher Ereignisse auf, so

24 Vgl. García Canclini, Néstor: Culturas híbridas. Estrategias para entrar y salir de la modernidad. Buenos Aires: Sudamericana 1992.

25 Zitiert nach Mignolo, Walter: Zur Frage der Schriftlichkeit in der Legitimation der Conquista. In: Kohut, Karl et al. (Hg.): Der eroberte Kontinent. Frankfurt am Main: Vervuert 1991, S. 87. 
dass er etwa vom Besuch einer japanischen Delegation in Neu-Spanien berichten konnte, welche im Übrigen die Eliten Neuspaniens erstmals mit japanischen „Biombos“, mit in Japan verfertigten Paravents vertraut machte. ${ }^{26}$ Die Geschichte dieser Paravents ist überaus spannend, wirft ein bezeichnendes Licht auf die Vielfalt der wirtschaftlichen wie der kulturellen Beziehungen zwischen Europa, Amerika und Asien und hatte letztlich - ganz nebenbei - zur Folge, dass Paravents im deutschen Sprachraum bis in unsere Zeit hinein nicht mit Hilfe eines französischen Ausdrucks, sondern als „Spanische Wand“ bezeichnet wurden.
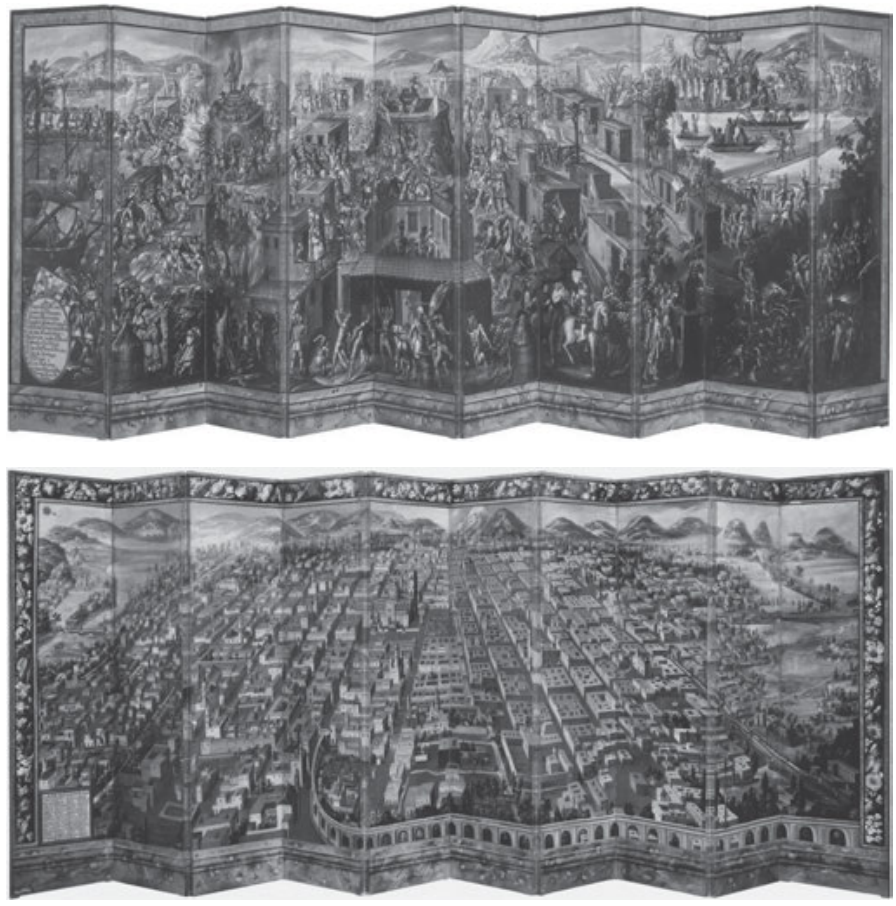

Abb. 28a und 28b: „Biombo de la Conquista y de la Muy Noble y Leal Ciudad de México“, circa 1690.

Ich möchte Ihnen an dieser Stelle unserer Vorlesung ein kleines Modell oder Schema an die Hand geben, das Ihnen erlauben soll, den weiten kulturellen Fächer unterschiedlicher Kulturen in Lateinamerika übersichtlicher zu struktu-

26 Vgl. hierzu Ette, Ottmar: Magic Screens. Biombos, Namban Art, the Art of Globalization and Education between China, Japan, India, Spanish America and Europe in the 17th and 18th Centuries. In: European Review (Cambridge) XXIV, 2 (May 2016), S. 285-296. 
rieren. Dabei geht es mir nicht darum, die kulturelle Komplexität der sogenannten ,Neuen Welt‘ für altweltliche Benutzerinnen und Benutzer zu reduzieren und gerade auch die unterschiedlichen Formen der Hybridisierung und Transkulturation zu überspielen. Wenn es gewiss auch notwendig wäre, regionale Besonderheiten Lateinamerikas stärker herauszuarbeiten, so lässt sich im Rahmen unserer Fragestellung die kulturelle Vielfalt in den so unterschiedlichen Areas der lateinamerikanischen Welt als Spannungsfeld darstellen, das von zumindest sechs verschiedenen kulturellen Polen geprägt ist:

1. Die vorbildgebende iberische Kultur im Kontext ihrer unterschiedlichen abendländischen Traditionsstränge.

2. Die sehr verschiedenartigen indigenen Kulturen, deren Fortbestehen im 19. Jahrhundert häufig geleugnet und deren Kulturraum ausgegrenzt wurde, die aber einen sich heute wieder erneuernden kulturellen Pol darstellen.

3. Die schwarzen Kulturen, die durch die koloniale Sklavenwirtschaft zwangsweise aus unterschiedlichen Kulturzonen Afrikas nach Amerika deportiert wurden und auf amerikanischem Boden reiche Formen der Transkulturation entwickelten.

4. Die iberischen Volkskulturen, welche die Eroberer mitgebracht hatten und die in unterschiedlichem Maße Eingang in ,hochkulturelle‘ Ausdrucksformen fanden.

5. Kulturelle Formen der Hybridisierung sowie transkulturelle Ausdrucksformen, die aus Kulturkontakten zwischen den vorher genannten Polen entstanden und die über lange Zeit von dem an Europa ausgerichteten städtischen Raum negiert und marginalisiert wurden.

6. Eine sich gegen Ende des 19. Jahrhunderts herausbildende Massenkultur, die sich nachfolgend im Zeichen einer sich verstärkenden Kulturindustrie einen immer weiteren Einflussbereich schuf und sich teilweise internationalisierte.

Die Ausdifferenzierung in sechs verschiedene Pole tritt an die Stelle einer einfachen Unterscheidung zwischen ,Volkskultur‘ und ,Hochkultur‘, welche der kulturellen Hybridisierung Lateinamerikas nicht in ausreichendem Maße gerecht wurde und eine notwendige Anpassung an sehr unterschiedliche regionale kulturelle Spannungsfelder nicht erlaubte. Insbesondere die indigenen und schwarzen Kulturen sind für weite Teile der Landbevölkerung, aber auch für städtische Randgruppen von großer Bedeutung; sie bilden einen über lange Zeit negierten, verdrängten Teil von Identitätszuschreibungen der sich an Europa orientierenden ,offiziellen' Literaturen. Es bedarf keiner weiteren Erläuterungen, dass es innerhalb der indigenen wie der schwarzen Kulturen weiterer Differenzierungen bedarf, um das in einer bestimmten Area wie etwa der Karibik oder dem Andenraum bestehende kulturelle Spannungsfeld präziser zu beschreiben. 
Es ist höchst aufschlussreich, dass sich bezüglich dieses Modells sechs verschiedener kultureller Pole in Lateinamerika die vielleicht wichtigsten und markantesten Unterschiede zwischen den einzelnen Vertretern des hispanoamerikanischen Modernismo gegen Ende des 19. Jahrhunderts zeigen. In einem ganz allgemeinen Sinne ließe sich ein Charakteristikum lateinamerikanischer Literaturen in der unterschiedlichen Herstellung von Beziehungen erblicken, welche den dominanten ersten Pol der abendländischen Kulturtradition mit anderen Polen des genannten Spannungsgefüges verbinden. Dabei geht es jedoch nicht allein um die jeweils besondere Art der Auseinandersetzung mit oder Integration von anderen kulturellen Polen in das je eigene Schreiben. Denn gerade das Ausblenden bestimmter kultureller Traditionen lässt sich als wichtiger und tiefgreifender Hinweis für die Deutung eines lateinamerikanischen Textes verstehen.

Während der Kolonialzeit lässt sich für den ersten Pol des skizzierten Spannungsfeldes eine Ausweitung des geokulturellen Raumes insoweit beobachten, als zunehmend philosophische oder literarische Einflüsse des nicht-iberischen Europa auf die Bildungseliten der lateinamerikanischen Städte einwirkten. Das sicherlich eindrücklichste Beispiel für diese Veränderungen ist der wachsende Einfluss, den die Schriften französischer Aufklärungsphilosophen im 18. Jahrhundert auf die kreolische Oberschicht in den Kolonien ausübten. Wir hatten gesehen, dass ein Autor wie Alejo Carpentier selbst am Beispiel Haitis in El reino de este mundo die Bedeutung der französischen Encyclopédie unterstrich, indem er sie im Inventar seines Herrenhauses auf der Plantage aufführte und sie danach in Flammen aufgehen ließ. Dass er dies auch für den spanischsprachigen Kolonialraum betonte, zeigte er an einer Vielzahl von Stellen im Roman El Siglo de las Luces auf.

Seit dem 18. Jahrhundert zeichnete sich in den iberischen Kolonien eine wachsende kulturelle Orientierung an Frankreich ab, welche man als eine Entwicklung hin zu einem geokulturellen Dominantenwechsel bezeichnen darf: Frankreich trat intellektuell an die Stelle Portugals und Spaniens, die aus Sicht der Kolonisten im Aufklärungszeitalter hinter Frankreich zurückblieben. Dieses Gefälle sollte sich im folgenden Jahrhundert noch wesentlich verstärken und Paris zur kulturellen Hauptstadt Lateinamerikas werden lassen. Die französische Metropole kann folglich mit Walter Benjamin mit Fug und Recht als „Hauptstadt des 19. Jahrhunderts“ angesehen werden, ${ }^{27}$ wenn man auch die lateinamerikanischen Nationen mit hinzudenkt. Die Literaturen Europas - und später die US-amerikanische Literatur - wurden in Lateinamerika zu keinem Zeitpunkt als homogener

27 Vgl. Benjamin, Walter: Paris, die Hauptstadt des XIX. Jahrhunderts. In (ders.): Das PassagenWerk. Bd. 1. Frankfurt am Main: suhrkamp 1983, S. 45-59. 
Block wahrgenommen. Vielmehr zeigten sich schon früh hinsichtlich der Bedeutung einzelner europäischer Nationalliteraturen auffällige Differenzen innerhalb unterschiedlicher kultureller Areas Lateinamerikas. Von lateinamerikanischer Seite aus wurde die Vielfalt der europäischen Literaturen immer anerkannt.

Die inter- und transkulturellen Bezüge zwischen Europa und Lateinamerika stellten, so dürfen wir festhalten, einen kulturellen Raum dar, der gemeinsam war (und es in gewisser Weise auch blieb), weil sich intensive Beziehungen seit Anfang des 16. Jahrhunderts entwickelten. Zugleich war (und ist) er widersprüchlich oder gegensätzlich, weil diese Beziehungen keinen egalitären Raum bilden, sondern sich innerhalb einer sich stark verändernden Asymmetrie ausbilden. Denn ganz wie im wirtschaftlichen, politischen oder sozialen Bereich sind diese Beziehungen während der Kolonialzeit zutiefst ungleich und verändern sich nach der Independencia nur graduell. Auch auf Ebene kultureller und literarischer Beziehungen sind beide Teilräume durch die Gegensätzlichkeit von europäischer Metropole und amerikanischer Peripherie gekennzeichnet, die sich dann im Verlauf des 20. Jahrhunderts langsam in ein neues System unterschiedlicher und mit einer jeweiligen Eigen-Logik ausgestatteter Literaturen der Welt veränderte. ${ }^{28}$ Das 19. Jahrhundert kann als eine Sattelzeit dieser komplexen Entwicklungen von der einen und ganz im Goethe'schen Sinne zentrierten Weltliteratur zu den vielen und viellogischen Literaturen der Welt angesehen werden.

Die (,offizielle‘, also am ersten Pol orientierte) kolonialspanische Literatur in Amerika entsteht aus der Übernahme und kreativen Verwendung literarischer Vorgaben, wobei hierunter nicht nur Einzeltexte zu verstehen sind, sondern auch Gattungen, die in Europa in einer bestimmten literarischen Tradition und Hierarchie verankert sind. Wir werden dies anhand unseres ersten Textbeispiels erkennen, etwa der Verwendung des Pikaresken in Fernández de Lizardis El Periquillo Sarniento, ein Roman, der ab 1816 in verschiedenen Lieferungen in einer Auflagenhöhe von circa fünfhundert Exemplaren in Mexiko erschien. Doch nicht nur im Bereich der erzählenden Prosa, sondern auch der Lyrik machten sich amerikanische Deutungen europäischer Modelle bemerkbar, die ein spezifisches Licht auf die jeweiligen Literaturbedingungen in unterschiedlichen Areas des kolonialen Amerika werfen. ${ }^{29}$

28 Vgl. hierzu Ette, Ottmar: Von den historischen Avantgarden bis nach der Postmoderne, S. 228234.

29 Vgl. hierzu Bernaschina Schürmann, Vicente: Angeles que cantan de continuo. Auge y caída de una legitimación teológica de la poesía culta en el virreinato del Perú. Potsdam: Universitätsverlag Potsdam 2019. 
Diese Überlegungen und Beispiele machen zum einen deutlich, dass Rückgriff und Aneignung ,fremder' literarischer Bezugstexte und Modelle keineswegs erst mit dem Ende des 19. Jahrhunderts oder mit der Independencia einsetzen. Zum anderen aber zeigt sich genau an diesem Punkt, dass eine noch immer verbreitete Vorstellung, der zufolge das Schreiben in Amerika weitgehend auf einer Imitation europäischer Vorgaben beruhe, schon für den kolonialspanischen Zeitraum -wie eine Vielzahl von Studien zeigte - viel zu kurz greift. Denn Begriffe wie Original und Kopie verlieren innerhalb des skizzierten widersprüchlichen kulturellen Raumes weitgehend ihren Erkenntnisgewinn und vermögen nicht zu erklären, wie vielfältig die kulturelle und literarische Produktion in den amerikanischen Kolonien tatsächlich war. Hier konnte die jüngere Forschung eine Reihe gerne geglaubter Vorurteile kassieren und unser Bild des kolonialen Amerika ungeheuer bereichern.

Denn die ,nachgeahmten' literarischen Formen stehen in den iberischen Kolonien in einem anderen kulturellen Spannungsfeld und erfüllen auch andere soziale Funktionen als in Europa. Der vielbemühte Pierre Menard, autor del Quijote von Jorge Luis Borges hat literarisch vorgeführt, dass ein mit Cervantes' Don Quijote textidentischer, aber später verfasster Roman in Fragmenten schon auf Grund seines veränderten historischen Kontexts ein anderer Text (geworden) ist. Dass eine solche Erkenntnis gerade im Bereich der lateinamerikanischen Literaturen ästhetisch überzeugend vorgeführt wurde, hängt mit der hier skizzierten Grundlegung des Schreibens seit der Kolonialzeit in wesentlicher Weise zusammen. Borges' Fiktion führt folglich auch für frühere, kolonialspanische Zeiten vor, dass eine Übersetzung - denn um eine solche handelt es sich ja bei dem Franzosen Pierre Menard - auch auf Ebene des Über-Setzens zwischen Alter und Neuer Welt neue Funktionen eröffnet und erfüllt. Die Überquerung des Atlantik, der Übergang von der einen auf die andere Seite der ,beiden Welten` veränderte damit über Funktion und Kontext die literarischen Formen selbst, die in Lateinamerika heimisch wurden - über Jahrhunderte freilich in einer Art und Weise, die keine literarischen Rückwirkungen auf die europäischen Literaturen zeitigte. Europa war an der Weiterentwicklung seiner literarischen Formen in Übersee weitestgehend desinteressiert. Dies sollte auch noch für einen guten Teil des 19. Jahrhunderts gelten.

Man könnte nun erwarten, dass die Erlangung politischer Unabhängigkeit der lateinamerikanischen Länder mit Ausnahme Kubas und Puerto Ricos im ersten Drittel des 19. Jahrhunderts zu einer einschneidenden Veränderung der skizzierten literarischen Beziehungen zwischen Ex-Metropole und Ex-Kolonie geführt hätte. Das war aber keineswegs der Fall und sollte uns davor warnen, leichtfüßig Ereignisse aus dem politischen Feld in das sich stärker autonomisierende literarische Feld zu übertragen. 
Die europäisch-lateinamerikanischen Literaturbeziehungen veränderten sich im Zeichen errungener politischer Unabhängigkeit nicht grundlegend und blieben auch weiterhin von einer deutlichen Asymmetrie geprägt. Wie der politischen Independencia keine wirtschaftliche entsprach, sondern vielmehr neue Abhängigkeitsbeziehungen entstanden und aufgebaut wurden, so ist auch im kulturellen und literarischen Bereich nicht von einer grundlegenden Umwälzung der Beziehungen zwischen Europa und Lateinamerika zu sprechen.

Gewiss traten nun Paris und in geringerem Maße London, ja selbst der deutschsprachige Raum ${ }^{30}$ an die Stelle der iberischen Hauptstädte als vorherrschende kulturelle Zentren, an denen sich die lateinamerikanischen Bildungseliten orientierten. Gewiss wurde die Parisreise für ungezählte lateinamerikanische Intellektuelle zum Initiationsritus und fand in literarisierter Form breiten Eingang in Romane und Reiseberichte des 19. Jahrhunderts: Wer etwas auf sich hielt, musste zumindest einmal für längere Zeit in der „ville-lumière“ gewesen sein. Doch haben wir es hier trotz aller Veränderungen im Grunde nur mit einem geokulturellen Dominantenwechsel zu tun: Literatur, Kunst und Kultur richteten sich in den lateinamerikanischen Ländern nun vorrangig an anderen Zentren aus, die jedoch noch immer in Europa lagen.

Lässt sich daher keine grundsätzliche Veränderung hinsichtlich des europäisch-lateinamerikanischen Wissenstransfers im 19. Jahrhundert beobachten, so zeigt sich dies auch bei der Untersuchung des Informationsflusses in umgekehrter Richtung. Denn in gewisser Weise sind nun die europäischen Reisenden an die Stelle der Chronisten getreten und liefern den wissenschaftlichen wie ökonomischen Zentren der europäischen Gesellschaften die primär für deren Interessen notwendigen Informationen aus Amerika. ${ }^{31}$ Es ist beeindruckend und zugleich für die fortbestehende intellektuelle Abhängigkeit charakteristisch, dass sich die kul-

30 Vgl. hierzu Krumpel, Heinz: Die deutsche Philosophie in Mexiko. Ein Beitrag zur interkulturellen Verständigung seit Alexander von Humboldt. Frankfurt am Main - Bern - New York: Peter Lang 1999; (ders.): Aufklärung und Romantik in Lateinamerika. Ein Beitrag zu Identität, Vergleich und Wechselwirkung zwischen lateinamerikanischen und europäischen Denkern. Frankfurt am Main Berlin - New York: Peter Lang 2004; (ders.): Barock und Moderne in Lateinamerika. ein Beitrag zu Identität und Vergleich zwischen lateinamerikanischem und europäischem Denken. Frankfurt am Main - Berlin - New York: Peter Lang 2008.

31 Vgl. speziell zur Rolle der britischen Reisenden Pratt, Mary Louise: Humboldt y la reinvención de América. In: Nuevo Texto Crítico (Stanford) 1 (1987), S. 35-53, sowie spezieller zu Humboldt Ette, Ottmar: „Unser Welteroberer“: Alexander von Humboldt, der zweite Entdecker, und die zweite Eroberung Amerikas. In: Amerika: 1492-1992. Neue Welten - Neue Wirklichkeiten. Essays. Herausgegeben vom Ibero-Amerikanischen Institut Preußischer Kulturbesitz und Museum für Völkerkunde Staatliche Museen zu Berlin. Braunschweig: Westermann 1992, S. 130-139. 
turelle und politische Elite der verschiedenen in Entstehung begriffenen Länder Lateinamerikas hinsichtlich der Beschaffenheit ihrer Staaten auf nicht immer wissenschaftlich abgesicherte europäische Quellen bezieht.

Zweifellos war es für die Literaturkritik wie die Literaturwissenschaft verlockend, nach kultureller oder literarischer Unabhängigkeit in Folge der Independencia $\mathrm{zu}$ fahnden. Gestützt auf die Selbstdarstellung führender Denker und Autoren des Modernismo wurde man hier auch bald fündig und glaubte, in der modernistischen Bewegung Hispanoamerikas endlich so etwas wie eine literarische Unabhängigkeitserklärung erkennen zu können. Doch scheint es mir verfehlt, den politischen Begriff der Unabhängigkeit auf die heteronome Sphäre des Kulturellen beziehungsweise Literarischen zu übertragen, ist die Vorstellung literarischer Unabhängigkeit und Autonomie angesichts der Vielfalt interkultureller Beziehungen doch ebenso absurd wie irreführend. Denn die Strukturen des politischen Feldes lassen sich nicht unverändert auf jene des literarischen Feldes übertragen, auch wenn die jeweiligen Macht-Eliten in Lateinamerika eine eng und gut verzahnte sowie überschaubare Gruppe innerhalb der Gesamtgesellschaften bildeten.

Vor dem Hintergrund der soeben vorgestellten Grundstrukturen literarischer Beziehungen zwischen Europa und Lateinamerika ist es möglich, gerade von dieser Fragestellung aus die große Bedeutung, die im letzten Drittel des 19. Jahrhunderts dem hispanoamerikanischen Modernismo in einem weltliterarischen Kontext zukommt, herauszuarbeiten. Dies führt mich zu einer These, die ich erst gegen Ende dieser Vorlesung anhand der Beschäftigung mit jenen Autoren überprüfen und erhärten kann, die über Lateinamerika hinaus gewirkt, vor allem aber die Grundbedingungen und Strukturen des Schreibens auf dem Subkontinent selbst verändert haben. Meine erst ganz am Schluss zu überprüfende These also lautet: Erst mit dem hispanoamerikanischen Modernismo setzt eine neue Phase literarischer Beziehungen zwischen Lateinamerika und Europa ein. Und mehr noch: Erst im letzten Drittel des 19. Jahrhunderts lassen sich die Anfänge dessen beobachten, was man einen wechselseitigen, also einen im vollen Wortsinne verstandenen Polylog auf Augenhöhe zwischen den Literaturen beiderseits des Atlantiks, also in beiden Welten, nennen könnte.

Denn erst seit diesem Zeitpunkt zeichnet sich eine weltliterarische Entwicklung ab, die ein Einwirken der lateinamerikanischen auf die europäischen Literaturen möglich machte, hatten sich doch die Rezeptionsbedingungen in Europa $\mathrm{zu}$ verändern begonnen. Nun wurden wechselseitige literarische Relationen denk- und implementierbar, welche über singuläre und rein punktuelle Kulturberührungen hinausgingen. Die zentrierten Vorstellungen von Weltliteratur gleichgültig, ob sie gedanklich in Weimar, Paris, London oder New York zusammenliefen - begannen sich zugunsten der Entfaltung jener Literaturen der Welt 
aufzulösen, die allesamt über ihre jeweiligen eigenen Logiken innerhalb eines viellogischen Gesamtsystems verfügen und eigene Charakteristika entwickeln, die von keinem Zentrum her mehr gebündelt werden können. ${ }^{32}$

Doch greifen wir dem Gang unserer Vorlesung nicht vor und wenden wir uns nunmehr aus amerikanischer Perspektive jenen Literaturen und literarischen Traditionen zu, welche zu Beginn des 19. Jahrhunderts die Beziehungen der Literaturen zwischen zwei Welten charakterisierten! Denn bis zum Erfolg der Autoren des sogenannten „Boom“ war es - literarhistorisch gesprochen - noch ein weiter Weg, und es sollte für uns keineswegs ein Weg sein, der direkt zu seinem Ziel führte, der ,Apotheose“ lateinamerikanischen Schreibens in den literarischen Zentren Europas und Nordamerikas, ja in weiten Teilen der literarischen Welt. Denn glücklicherweise führen nicht alle Wege zum Boom: Der Subkontinent ist literarisch viel reicher und vielfältiger.

Nicht nur die kolonialspanische Literatur verdient eine substanzielle Aufwertung, welche bereits - wie erwähnt - in vollem Gange ist, sondern auch das 19. Jahrhundert mit seinen Literaturen zwischen den Welten. Ist die gegenwärtige lateinamerikanische Literatur - wie Jean Franco ${ }^{33}$ noch Mitte der siebziger Jahre des vergangenen Jahrhunderts schrieb - wirklich die wichtigste lateinamerikanische Literatur überhaupt? Ist es daher gerechtfertigt, sich auf die Literaturen des 20. Jahrhunderts zu konzentrieren und dabei die Literaturen des 19. Jahrhunderts weniger ausführlich zu behandeln, ja vielleicht nur zu überfliegen. Ich bin, wie Sie mit Recht vermuten, nicht dieser Ansicht. Und der weitere Fortgang unserer Vorlesung soll diese Ansicht untermauern!

32 Vgl. hierzu Ette, Ottmar: Die Literaturen der Welt. Transkulturelle Bedingungen und polylogische Herausforderungen eines prospektiven Konzepts. In: Lamping, Dieter / Tihanov, Galin (Hg.): Vergleichende Weltliteraturen / Comparative World Literatures. DFG-Symposion 2018. Unter Mitwirkung von Mathias Bormuth. Stuttgart: J.B. Metzler - Springer 2019, S. 115-130.

33 Franco, Jean: Historia, S. 12. 